\title{
Gaining Strength For A New Future: Bosnia And Herzegovina's Export Opportunities
}

Emin Civi, University of New Brunswick, Saint John, Canada

Elif S. Persinger, (E-mail: esonmez@emich,edu), University of Eastern Michigan, USA

Aziz Sunje, University of Sarajevo, Bosnia and Herzegovina

\begin{abstract}
International trade is crucial for Bosnia and Herzegovina's (B\&H) economic prosperity. In this study guidance to $B \& H$ exporters is provided by identifying potential markets and products to focus on when designing future trade strategies. To this end trends in the world trade and trade patterns are examined using various approaches.

First approach to identify the potential markets for the $B \& H$ exports called for identifying the countries with highest general demand for Bosnia and Herzegovina's current export products. The products Bosnia and Herzegovina exports most along with the countries that demand these products the most in the world are identified. The second approach for identifying the potential export markets for B\&H products examine the import volumes of other countries in the world. Still a more fruitful approach for the B\&H exporters, at least in the short term, is to target the markets with the fastest growth of import volumes (the third approach). In the fourth approach, "untapped trade" and "highly untapped trade" countries that should be targeted by B\&H exporters are presented. The fifth approach for identifying the potential export markets for B\&H products is based on examining the products whose imports increased fastest in recent years and the countries that imported these products most.
\end{abstract}

The products/product groups that have the highest potential for B\&H export success are also identified. First, most imported products as well as the products/product groups whose exports increased the fastest in recent years are examined. Second, the import volumes ten countries with the highest total imports are examined on a product basis to identify the products they import most as well as the products with the highest growth rate of imports. Third, product categories with "untapped trade potential" and "highly untapped trade potential" along with their respective markets are presented.

Long term sustainable success in the ever changing global economy requires a close and continuous scanning of the trends in the environment. The analysis approaches described above provide B\&H exporters a starting point in evaluating their product and market selection strategies and designing new ones for the future.

\section{INTRODUCTION}

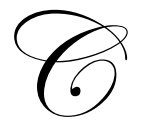

ountries strive to achieve economic prosperity in order to sustain their viability in the highly competitive economic arena of the $21^{\text {st }}$ century. Today's developed countries achieved the goal of economic prosperity for the most part through international trade. International trade is a component of globalization trend and an undeniable part of these countries' general economic policies. The increasing interdependence of socio-economic relationships among countries around the world no longer leaves room for application of inward-looking economic policies based on local industrial and economic advantages. 
This study consists of four parts. The first part describes the recent trends and the structural changes in the world trade. The second part includes an analysis of the economy of Bosnia and Herzegovina (B\&H) in general as well as in terms of export readiness. The third part is on the selection of markets that would be most profitable for $\mathrm{B} \& \mathrm{H}$ exporters. The fourth part addresses the issue of selection of products that can be most successful in these markets as well as the recommendations on policies and strategies for the exporters and the government to ensure this success.

\section{The Developments in the World Trade}

Both world production and world trade has grown rapidly in the recent decades. In fact, between 1995 and 2004, with the exception of 2001, world exports have grown faster than world output. Over these 10 years, world output has grown an average of $2.7 \%$ annually whereas world exports have grown an average of $5.9 \%$ annually. Trade growth continued to exceed output growth in 2004 (WTO 2005). This fact alone confirms the increasing importance of international trade for the economic policies of countries.

Table 1: World GDP and Merchandise Exports, 1995-2004 (Annual \% Change)

\begin{tabular}{|l|c|c|c|c|c|}
\hline & $\mathbf{1 9 9 5 - 0 0}$ & $\mathbf{2 0 0 1}$ & $\mathbf{2 0 0 2}$ & $\mathbf{2 0 0 3}$ & $\mathbf{2 0 0 4}$ \\
\hline World merchandise exports & 7.0 & -0.5 & 3.5 & 5.0 & 9.0 \\
\hline Agricultural products & 3.5 & 2.5 & 3.5 & 3.5 & 3.5 \\
\hline Fuels and mining products & 3.5 & 1.0 & 1.0 & 4.5 & 5.5 \\
\hline Manufactures & 8.0 & -1.0 & 4.0 & 5.0 & 10.0 \\
\hline World merchandise production & 4.0 & -0.5 & 2.5 & 3.5 & 4.0 \\
\hline Agriculture & 2.5 & 1.5 & 1.5 & 2.5 & 3.0 \\
\hline Mining & 2.0 & -0.5 & 0.0 & 3.5 & 4.0 \\
\hline Manufacturing & 4.0 & -1.0 & 3.0 & 3.5 & 4.0 \\
\hline World GDP & 3.0 & 1.0 & 1.5 & 2.5 & 3.5 \\
\hline
\end{tabular}

Source: WTO (2004), World Trade Report 2004: Exploring the Linkage between the Domestic Policy Environment and International Trade, WTO, Geneva and WTO (2005), World Trade Report 2005. Exploring the Links between Trade, Standards and the WTO, Geneva

Globalization of the world economy brought about not only faster growth of world economies and world trade but also increasing interdependence among countries which means that the effects of an economic crisis in one country is felt deeply in other countries as well. In 2001, the global impacts of such a crisis were slower economic growth and decreasing exports in the world.

According to the WTO statistics (2005), trade of goods in the world increased by $10 \%$ in 2004 . Although this represents a better performance compared to 2002 and 2003, it is still significantly lower than the growth in the second half of the 1990s. The positive change in the world trade compared to 2002 and 2003 is mainly due to the performances of the transition economies of Asia and Eastern Europe. The imports and exports of these two regions in 2004 grew by almost $15 \%$. As seen in Table 2, North American countries were close to the world average but did not contribute the world trade as much as the Asian and Eastern European countries. The exports of Western Europe, although somewhat recovered in 2004, increased by only $0.5 \%$ in 2003 and their imports grew only $1.5 \%$ in the same period. According to the World Trade Report (WTO 2004), this slow growth of trade in Western Europe is due to the slowdown of investments and consumer spending in this region. Same report also points to the increasing fiscal deficit in the USA (4.9\% of its GDP), Japan (7.4\% of its GDP) and European Union (2.7\% of its GDP) as one reason why global economy and hence world trade experienced accelerated growth in 2003. 
Table 2: World Merchandise Trade by Regions, 1995-04

(Annual \% Change)

\begin{tabular}{|c|c|c|c|l|c|c|c|c|}
\hline \multicolumn{4}{|c|}{ Exports } & \multirow{2}{*}{ Region } & \multicolumn{4}{c|}{ Imports } \\
\cline { 1 - 6 } $\mathbf{1 9 9 5 - 0 0}$ & $\mathbf{2 0 0 2}$ & $\mathbf{2 0 0 3}$ & $\mathbf{2 0 0 4}$ & & $\mathbf{1 9 9 5 - 0 0}$ & $\mathbf{2 0 0 2}$ & $\mathbf{2 0 0 3}$ & $\mathbf{2 0 0 4}$ \\
\hline 7.0 & 3.0 & 4.5 & 9.0 & World & 7.0 & 3.0 & 5.0 & 10.0 \\
\hline 7.0 & -2.5 & 1.5 & 7.5 & North America & 10.5 & 4.0 & 5.5 & 10.5 \\
\hline 9.5 & -0.5 & 4.0 & 13.0 & Latin America & 10.0 & -7.0 & 0.5 & 17.5 \\
\hline 6.0 & 1.5 & 0.5 & 6.5 & Western Europe & 6.0 & 0.5 & 1.5 & 6.5 \\
\hline 7.0 & 8.0 & 12.5 & 13.0 & C./E. Europe/Baltic States/CIS & 7.5 & 7.5 & 11.5 & 15.0 \\
\hline 8.5 & 10.5 & 12.0 & 14.0 & Asia & 5.5 & 8.5 & 11.0 & 14.5 \\
\hline 4.5 & 8.0 & 5.0 & 10.5 & Japan & 4.5 & 1.5 & 7.0 & 7.0 \\
\hline 9.5 & 8.0 & 9.5 & 14.5 & Six East Asian Traders & 4.0 & 6.5 & 5.0 & 14.5 \\
\hline
\end{tabular}

Source: WTO (2004), World Trade Report 2004: Exploring the Linkage Between the Domestic Policy Environment and International Trade, WTO, Geneva and WTO (2005), World Trade Report 2005. Exploring the Links Between Trade, Standards and the WTO, Geneva

Table 3 (as well as Table 2) shows that developing countries, particularly those in Asia and Eastern Europe, have been increasing their share in world trade in the recent decades. The trade growth attributed to these countries not only fueled the dynamism in the world trade but also contributed to their competitiveness in the global markets, enhancing their efforts toward the mission to take place among the developed nations of the world.

Table 3: Leading Exporter and Importers in World Trade, 2004 (Billion Dollars, \% of World Trade, Annual \% Change)

\begin{tabular}{|c|c|c|c|c|c|c|c|c|c|}
\hline Rank & Exporter Country & Value & $\%$ & $\begin{array}{c}\text { Annual } \\
\text { Change, \% }\end{array}$ & Rank & Importing Country & Value & $\%$ & $\begin{array}{c}\text { Annual } \\
\text { Change, \% }\end{array}$ \\
\hline 1 & Germany & 912.3 & 10.0 & 21 & 1 & United States & 1525.5 & 16.1 & 17 \\
\hline 2 & United States & 818.8 & 8.9 & 13 & 2 & Germany & 716.9 & 7.6 & 19 \\
\hline 3 & China & 593.3 & 6.5 & 35 & 3 & China & 561.2 & 5.9 & 36 \\
\hline 4 & Japan & 565.8 & 6.2 & 20 & 4 & France & 465.5 & 4.9 & 17 \\
\hline 5 & France & 448.7 & 4.9 & 14 & 5 & United Kingdom & 463.5 & 4.9 & 18 \\
\hline 6 & Netherlands & 358.2 & 3.9 & 21 & 6 & Japan & 454.5 & 4.8 & 19 \\
\hline 7 & Italy & 349.2 & 3.8 & 17 & 7 & Italy & 351.0 & 3.7 & 18 \\
\hline 8 & United Kingdom & 346.9 & 3.8 & 13 & 8 & Netherlands & 319.3 & 3.4 & 21 \\
\hline 9 & Canada & 316.5 & 3.5 & 16 & 9 & Belgium & 285.5 & 3.0 & 22 \\
\hline 10 & Belgium & 306.5 & 3.3 & 20 & 10 & Canada & 279.8 & 2.9 & 14 \\
\hline 11 & Hong Kong & 265.5 & 2.9 & 16 & 11 & Hong Kong & 272.9 & 2.9 & 17 \\
\hline 12 & South Korea & 253.8 & 2.8 & 31 & 12 & Spain & 249.3 & 2.6 & 20 \\
\hline 13 & Mexico & 189.1 & 2.1 & 14 & 13 & South Korea & 224.5 & 2.4 & 26 \\
\hline 14 & Russian Federation & 183.5 & 2.0 & 35 & 14 & Mexico & 206.4 & 2.2 & 16 \\
\hline 15 & Taipei, Chinese & 182.4 & 2.0 & 21 & 15 & Taipei, Chinese & 168.4 & 1.8 & 32 \\
\hline 16 & Singapore & 179.6 & 2.0 & 25 & 16 & Singapore & 163.9 & 1.7 & 28 \\
\hline 17 & Spain & 178.6 & 2.0 & 14 & 17 & Austria & 117.8 & 1.2 & 18 \\
\hline 18 & Malaysia & 126.5 & 1.4 & 21 & 18 & Switzerland & 111.6 & 1.2 & 16 \\
\hline 19 & Saudi Arabia & 126.2 & 1.4 & 35 & 19 & Australia & 109.4 & 1.2 & 23 \\
\hline 20 & Sweden & 122.5 & 1.3 & 20 & 20 & Malaysia & 105.3 & 1.1 & 26 \\
\hline 21 & Switzerland & 118.5 & 1.3 & 18 & 21 & Sweden & 99.3 & 1.0 & 19 \\
\hline 22 & Austria & 117.4 & 1.3 & 21 & 22 & Turkey & 97.5 & 1.0 & 41 \\
\hline 23 & Ireland & 104.3 & 1.1 & 12 & 23 & India & 97.3 & 1.0 & 37 \\
\hline 24 & Thailand & 97.4 & 1.1 & 21 & 24 & Russian Federation & 96.3 & 1.0 & 27 \\
\hline 25 & Brazil & 96.5 & 1.1 & 32 & 25 & Thailand & 95.4 & 1.0 & 26 \\
\hline
\end{tabular}

WTO (2004), World Trade Report 2004: Exploring the Linkage Between the Domestic Policy Environment and International Trade, WTO, Geneva 


\section{The Economy of Bosnia and Herzegovina}

Bosnia and Herzegovina (B\&H) is among the developing countries that are seen as rising stars of the world trade due to their growing share. Despite its rapid economic growth recently, B\&H has been struggling to transform its economic, social and political systems since the Dayton Peace Accords that concluded the 1992-95 civil war. Before the war, B\&H was a medium-developed industrial and agricultural republic within the former State of Yugoslavia, a centrally planned economy, with about 1,000 greatly overstaffed industrial organizations (UNDP, 2003). Since the end of the war, B\&H has seen a remarkable transformation of its devastated society and economy. The IMF estimated that the real GDP growth in B\&H in 2005 was 5\%, lower than the $15.5 \%$ peak in 1998 but still quite impressive. The GDP per capita was estimated just over USD 2,000, slightly over its pre-war (1991) level of USD 1,979. The country's critical infrastructure is rebuilt, and modern political and economic institutions and processes are established due to the combined efforts of the B\&H government, international financial institutions such as the World Bank (WB) and the International Monetary Fund (IMF), international donors and limited foreign investment. B\&H started its process of accession to the World Trade Organization (WTO) in July 1999 by obtaining Observer status. Since then, B\&H puts all its efforts to adjust existing legislation and to adopting all necessary legislation in accordance with WTO rules, provisions and regulations (World Bank, 2000).

The post-war economy of B\&H is heavily dependent on imports mostly from the neighboring countries of Croatia, Yugoslavia, Slovenia, Austria and Germany. Domestic production with a few minor exceptions is limited. As B\&H's trade routes to Europe did not run through Yugoslavia, exports were not disrupted by the bombing and sanctions during the Kosovo campaign in 1999 to the same extent as some other Balkan states (Link2Exports, 2007). Recent strong growth in imports of investment goods helps strengthening the country's export base, and significant export-oriented capacity has come on stream in the past year. Along with rising demand in several of B\&H's leading export markets, this should bring about robust export growth in the coming years.

One of the main obstacles to B\&H's efforts to increase the competitiveness of its economy is state-owned enterprises that represent as much as $60 \%$ of the economy. In fact, many firms (both privatized and state-owned) do not seek profits, loss-making is widespread and workers tolerate overdue in wage. One of the urgent remedies to make the economy more competitive is to support establishment of private enterprises. However, B\&H does not provide a very convenient environment for starting new businesses ${ }^{1}$ (World Bank, 2005). The challenges of launching a business in B\&H are shown in Table 4. Entrepreneurs can expect to go through 12 steps to launch a business over 54 days on average, at a cost equal to $40.9 \%$ of gross national income (GNI) per capita. They must deposit at least $57.4 \%$ of GNI per capita in a bank to obtain a business registration number. To start new business is relatively expensive in B\&H compared to the OECD countries and former Yugoslavian countries (The World Bank, 2005).

\footnotetext{
${ }^{1}$ Starting a business topic identifies the bureaucratic and legal hurdles an entrepreneur must overcome to incorporate and register a new firm. It examines the procedures, time, and cost involved in launching a commercial or industrial firm with up to 50 employees and start-up capital of 10 times the economy's per-capita gross national income (GNI).
} 
Table 4: Starting a Business

\begin{tabular}{|c|c|c|c|c|}
\hline Region/Economy & $\begin{array}{c}\text { Procedures } \\
\text { (number) }\end{array}$ & Duration (days) & $\begin{array}{c}\text { Cost } \\
(\% \text { GNI per capita) }\end{array}$ & $\begin{array}{c}\text { Min. Capital } \\
\text { (\% GNI per capita) }\end{array}$ \\
\hline Bosnia and Herzegovina & 12 & 54 & 40.9 & 57.4 \\
\hline East Asia \& Pacific & 8.2 & 52.6 & 42.9 & 109.2 \\
\hline Europe \& Central Asia & 9.6 & 36.4 & 13.5 & 49.1 \\
\hline Latin America \& Caribbean & 11.4 & 63 & 56.2 & 24.1 \\
\hline Middle East \& North Africa & 10.1 & 45.4 & 64.2 & 859.3 \\
\hline OECD & 6.5 & 19.5 & 6.8 & 41 \\
\hline South Asia & 7.9 & 35.3 & 40.5 & 0.8 \\
\hline Sub-Saharan Africa & 11 & 63.8 & 215.3 & 297.2 \\
\hline Albania & 11 & 41 & 31.1 & 39.9 \\
\hline Argentina & 15 & 32 & 13.4 & 6.6 \\
\hline Austria & 9 & 29 & 5.7 & 61.5 \\
\hline Brazil & 17 & 152 & 10.1 & 0 \\
\hline Canada & 2 & 3 & 0.9 & 0 \\
\hline China & 13 & 48 & 13.6 & 946.7 \\
\hline Croatia & 12 & 49 & 13.4 & 22.7 \\
\hline Czech Republic & 10 & 40 & 9.5 & 39 \\
\hline Egypt & 10 & 34 & 104.9 & 739.8 \\
\hline Estonia & 6 & 35 & 6.2 & 41.4 \\
\hline Ethiopia & 7 & 32 & 65.1 & $1,532.00$ \\
\hline India & 11 & 71 & 61.7 & 0 \\
\hline Italy & 9 & 13 & 15.7 & 10.8 \\
\hline Malaysia & 9 & 30 & 20.9 & 0 \\
\hline Romania & 5 & 11 & 5.3 & 0 \\
\hline Russia & 8 & 33 & 5 & 4.4 \\
\hline Serbia and Montenegro & 10 & 15 & 6 & 9.5 \\
\hline Slovenia & 9 & 60 & 10.1 & 17 \\
\hline Turkey & 8 & 9 & 27.7 & 20.9 \\
\hline United States & 5 & 5 & 0.5 & 0 \\
\hline
\end{tabular}

Source: The World bank (2005), Doing Business in 2006, Doing Business in 2006: Creating Jobs, World Bank, Washington DC.

The liberalization of trade in the world is one of the major priorities of all the countries including $\mathrm{B} \& \mathrm{H}$. B\&H's economy is highly trade dependent. Export growth is the main driving force of its economic development agenda. The country's major trade policy objective is the integration of national economy into the global economy. As a small country, B\&H is strongly interested in maintaining a well-functioning multilateral trading system, which is helping its companies get involved in the world market through export and direct investments. The state is supporting exporting companies by reducing the bureaucratic barriers over international trade. Table 5 shows that $\mathrm{B} \& \mathrm{H}$ is among the countries with short clearance time for exports and imports. 
Table 5: Trade and Bureaucracy- 2005

\begin{tabular}{|c|c|c|c|c|c|}
\hline Country/Region & $\begin{array}{l}\text { Average time } \\
\text { to clear direct } \\
\text { exports } \\
\text { through } \\
\text { customs } \\
\text { (days) }\end{array}$ & $\begin{array}{l}\text { Longest time } \\
\text { to clear direct } \\
\text { exports } \\
\text { through } \\
\text { customs } \\
\text { (days) }\end{array}$ & $\begin{array}{l}\text { Average time } \\
\text { to claim } \\
\text { imports from } \\
\text { customs } \\
\text { (days) }\end{array}$ & $\begin{array}{l}\text { Longest time } \\
\text { to claim } \\
\text { imports from } \\
\text { customs } \\
\text { (days) }\end{array}$ & $\begin{array}{c}\text { Firms that } \\
\text { export } \\
\text { directly }(\%)\end{array}$ \\
\hline Bosnia and Herzegovina & 2.00 & 2.65 & 2.47 & 3.82 & 32.00 \\
\hline East Asia \& Pacific & 3.75 & 5.99 & 5.39 & 9.81 & 32.32 \\
\hline Europe \& Central Asia & 3.26 & 6.10 & 3.85 & 7.82 & 24.16 \\
\hline Latin America \& Caribbean & 3.48 & 6.68 & 8.14 & 16.64 & 36.32 \\
\hline Middle East \& North Africa & 4.88 & 8.29 & 10.78 & 22.24 & 24.96 \\
\hline OECD & 4.63 & 8.33 & 5.28 & 9.40 & 20.01 \\
\hline South Asia & 6.25 & 10.49 & 9.46 & 17.28 & 28.99 \\
\hline Sub-Saharan Africa & 4.40 & 8.43 & 8.22 & 16.46 & 31.32 \\
\hline Albania (2005) & 1.36 & 2.68 & 1.70 & 3.90 & 33.33 \\
\hline Brazil (2003) & 7.85 & 16.35 & 12.41 & 30.58 & 27.08 \\
\hline Bulgaria (2005) & 1.67 & 2.97 & 2.41 & 4.65 & 22.00 \\
\hline China (2003) & 6.16 & 5.78 & 7.60 & 10.48 & 17.77 \\
\hline Croatia (2005) & 2.03 & 4.36 & 3.03 & 4.63 & 33.62 \\
\hline Ireland (2005) & 2.62 & 5.76 & 3.01 & 6.33 & 31.46 \\
\hline Macedonia, FYR (2005) & 1.91 & 3.60 & 3.10 & 6.65 & 25.00 \\
\hline Romania (2005) & 2.14 & 3.83 & 2.62 & 4.78 & 20.50 \\
\hline Russia (2005) & 7.18 & 13.61 & 7.12 & 17.6 & 13.81 \\
\hline Senegal (2003) & 5.88 & 10.21 & 5.60 & 12.36 & 41.91 \\
\hline Serbia and Montenegro (2005) & 3.02 & 5.28 & 4.72 & 9.29 & 31.33 \\
\hline Slovak Republic (2005) & 5.78 & 11.1 & 3.45 & 6.23 & 33.95 \\
\hline Slovenia (2005) & 2.85 & 5.47 & 2.83 & 7.17 & 42.53 \\
\hline Turkey (2005) & 4.20 & 5.40 & 5.62 & 9.17 & 35.91 \\
\hline
\end{tabular}

Source: The World Bank (2006), Enterprise Survey,

http://rru.worldbank.org/EnterpriseSurveys/ExploreEconomies/Default.aspx?economyid=26

\section{Potential Markets for B\&H Exporters}

Five different approaches are used to identify the potential markets for B\&H exporters. The first approach is based on the assumption that the countries that are already demanding B\&H products will continue to do so, at least in the near future. B\&H exporters should continue paying attention to these existing markets for their products since keeping or gaining ground in these markets is easier than penetrating new markets. Hence, as part of a low risk strategy, they cannot afford to let these markets slip away from them. Naturally, this approach does not undermine the importance of concentrating in totally new markets for B\&H products. In addition to existing export destination, B\&H should target countries which import these products most in the word market. The exports of B\&H products during the 2003-2004 period are analyzed to identify the products that are exported most and the countries that imported these products most. The results are given in Table 6. 


\section{Table 6: Major Exports by B\&H and Their Destinations}

\begin{tabular}{|c|c|}
\hline Product & Target Markets \\
\hline o84 - Aluminum & $\begin{array}{l}\text { USA, Germany, Japan, China, Italy, France, Korea Rep., Belgium, Canada, UK, Netherlands, Mexico, Spain, } \\
\text { Austria, Poland, Hungary, Switzerland, Turkey, Malaysia, Thailand, Czech Rep. }\end{array}$ \\
\hline 248 - Wood Simply Worked & $\begin{array}{l}\text { USA, Japan, UK, Italy, China, Germany, France, Spain, Netherlands, Canada, Belgium, Denmark, Mexico, } \\
\text { Australia, Austria, Egypt, Hong Kong, Norway, Ireland, Thailand, }\end{array}$ \\
\hline 821 - Furniture/Stuff Furnishing & $\begin{array}{l}\text { USA, Germany, UK, France, Japan, Canada, Belgium, Netherlands, Spain, Switzerland, Austria, Italy, } \\
\text { Sweden, Australia, Mexico, Norway, Hong Kong, Denmark, Poland, Czech Rep., }\end{array}$ \\
\hline 713 - Internal Combust Engines & $\begin{array}{l}\text { USA, Germany, Canada, France, UK, Spain, Mexico, Belgium, China, Italy, Hungary, Austria, Turkey, } \\
\text { Poland, Japan, Sweden, Korea Rep., Netherlands, Australia, Brazil }\end{array}$ \\
\hline 351 - Electric Current & $\begin{array}{l}\text { Austria, Italy, USA, Germany, France, Canada, Netherlands, UK, Belgium, Hong Kong, Norway, Sweden, } \\
\text { Spain, Hungary, Finland, Denmark, Slovakia, Argentina, Portugal, Slovenia }\end{array}$ \\
\hline 851 - Footwear & $\begin{array}{l}\text { USA, Germany, Hong Kong, UK, France, Italy, Japan, Belgium, Spain, Netherlands, Canada, Austria, } \\
\text { Switzerland, Australia, Denmark, Korea Rep., Sweden, Greece, China, Portugal }\end{array}$ \\
\hline 285 - Aluminum Ores/Concs/Etc & $\begin{array}{l}\text { China, Russian Fed., Canada, USA, Norway, South Africa, Germany, France, Italy, Netherlands, Egypt, New } \\
\text { Zealand, Spain, Bahrain, Argentina, Ukraine, Ireland, Iceland, Japan, Romania }\end{array}$ \\
\hline 841 - Men’s/Boys Wear, Woven & $\begin{array}{l}\text { USA, Germany, Japan, UK, France, Italy, Hong Kong, Spain, Belgium, Netherlands, Switzerland, Canada, } \\
\text { Korea Rep., Austria, Denmark, Sweden, Australia, Mexico, }\end{array}$ \\
\hline 211 - Hide/Skin (Ex Fur) Raw & $\begin{array}{l}\text { China, Italy, Korea Rep., Turkey, Hong Kong, Japan, Thailand, Mexico, Germany, Spain, Netherlands, } \\
\text { Austria, France, UK, USA, Moldova, India, Poland, Slovakia, Belgium }\end{array}$ \\
\hline 691 - Iron/Stl/Alum Structures & $\begin{array}{l}\text { USA, Germany, France, UK, Japan, Switzerland, Austria, Norway, Belgium, Netherlands, Spain, Canada, } \\
\text { Italy, Denmark, China, Russian Fed., Hong Kong, Poland, Ireland, Czech Rep. }\end{array}$ \\
\hline 282 - Ferrous Waste/Scrap & $\begin{array}{l}\text { Turkey, Korea Rep., China, Spain, Belgium, Germany, Italy, USA, India, Netherlands, France, Finland, } \\
\text { Luxembourg, Malaysia, Mexico, Indonesia, Japan, Greece, Sweden }\end{array}$ \\
\hline 325 - Coke/Semi-Coke/Retort C & $\begin{array}{l}\text { Germany, USA, Japan, India, Brazil, France, Italy, Romania, UK, Belgium, Korea Rep., Serbia and Mtneg., } \\
\text { Kazakhstan, Sweden, Czech Rep., Canada, Mexico, South Africa, Austria, Finland }\end{array}$ \\
\hline 288 - Nf Base Metal Waste Nes & $\begin{array}{l}\text { China, Germany, USA, Japan, UK, Belgium, Italy, Korea Rep., Canada, Netherlands, France, India, Austria, } \\
\text { Spain, Sweden, Luxembourg, Norway, Mexico, Hong Kong, Turkey }\end{array}$ \\
\hline 784 - Motor Veh Parts/Access & $\begin{array}{l}\text { USA, Canada, Germany, Spain, UK, France, Belgium, Mexi } \\
\text { Japan, Turkey, Poland, Czech Rep., Thailand, Slovakia, Kor }\end{array}$ \\
\hline 893 - Articles Nes Of Plastics & $\begin{array}{l}\text { USA, Germany, Mexico, France, UK, Japan, Canada, Belgit } \\
\text { Spain, Austria, Switzerland, Czech Rep., Poland, Sweden, T }\end{array}$ \\
\hline 699 - Base Metal Manufac Nes & $\begin{array}{l}\text { USA, Germany, Mexico, France, UK, Canada, Japan, China, Spain, It } \\
\text { Austria, Czech Rep., Thailand, Korea Rep., Sweden, Hong Kong, Hur }\end{array}$ \\
\hline 641 - Paper/Paperboard & $\begin{array}{l}\text { USA, Germany, UK, France, Italy, China, Spain, Belgium, N } \\
\text { Australia, Hong Kong, Austria, Turkey, Switzerland, Denma }\end{array}$ \\
\hline 676 - Iron/Steel Bars/Rods/Etc & $\begin{array}{l}\text { USA, Germany, France, Italy, Korea Rep., UK, Canada, Spain, Netherlands, Belgium, China, Mexico, } \\
\text { Portugal, Switzerland, Sweden, Hong Kong, Austria, Singapore, Algeria, Iran }\end{array}$ \\
\hline 671 - Pig Iron Etc Ferro Alloy & $\begin{array}{l}\text { USA, Japan, Korea Rep., Germany, Italy, China, Spain, Belgium, France, Netherlands, Finland, Malaysia, } \\
\text { Turkey, UK, Mexico, Russian Fed., Canada, Sweden, Poland, Czech Rep. }\end{array}$ \\
\hline 842 - Women/Girl Clothing Wven & $\begin{array}{l}\text { USA, Germany, Japan, UK, France, Hong Kong, Italy, Spain, Belgium, Netherlands, Switzerland, Canada, } \\
\text { Austria, Denmark, Korea Rep., Australia, Sweden, Mexico }\end{array}$ \\
\hline 635 - Wood Manufactures N.E.S. & $\begin{array}{l}\text { USA, Germany, Japan, UK, France, Italy, Belgium, Spain, Canada, Switzerland, Netherlands, Austria, } \\
\text { Denmark, Norway, Hong Kong, Sweden, Australia, Ireland, Czech Rep., Mexico }\end{array}$ \\
\hline 322 - Briquettes/Lignite/Peat & $\begin{array}{l}\text { USA, France, Netherlands, Italy, UK, Belgium, Austria, Germany, Japan, Spain, Slovenia, Slovakia, } \\
\text { Hungary, Sweden, Switzerland, Denmark, Serbia and Mtneg., }\end{array}$ \\
\hline 634 - Veneer/Plywood/Etc & $\begin{array}{l}\text { USA, Japan, UK, Germany, Italy, France, China, Netherlands, Canada, Spain, Belgium, Korea Rep., Mexico, } \\
\text { Poland, Denmark, Sweden, Austria, Switzerland, Turkey, Romania }\end{array}$ \\
\hline 845 - Articles Of Apparel Nes & $\begin{array}{l}\text { USA, Germany, Japan, UK, Hong Kong, France, Italy, Spain, Belgium, Netherlands, Canada, Switzerland, } \\
\text { Austria, Denmark, Mexico, Sweden, Singapore, Australia, Korea Rep., Norway }\end{array}$ \\
\hline 693 - Wire Prod Exc Ins Electr & $\begin{array}{l}\text { USA, France, Germany, Spain, UK, Belgium, China, Italy, Canada, Austria, Mexico, Netherlands, Japan, } \\
\text { Poland, Czech Rep., Denmark, Australia, Hungary, Singapore, Switzerland }\end{array}$ \\
\hline 891 - Arms And Ammunition & $\begin{array}{l}\text { USA, Korea Rep., Canada, Saudi Arabia, Cote Divoire, Australia, Switzerland, Japan, New Zealand, UK, } \\
\text { Spain, Turkey, Netherlands, France, Germany, Norway, Finland, }\end{array}$ \\
\hline 781 - Passenger Cars Etc & $\begin{array}{l}\text { USA, Germany, UK, Italy, France, Spain, Belgium, Canada, Netherlands, Japan, Australia, Austria, Mexico, } \\
\text { Switzerland, Russian Fed., Sweden, China, Turkey, Greece, Poland }\end{array}$ \\
\hline 247 - Wood In Rough/Squared & $\begin{array}{l}\text { China, Japan, India, Korea Rep., Finland, Austria, Sweden, Italy, Canada, France, Spain, USA, Germany, } \\
\text { Turkey, Norway, Belgium, UK, Viet Nam, Portugal, Thailand }\end{array}$ \\
\hline 743 - Fans/Filters/Gas Pumps & $\begin{array}{l}\text { USA, China, Germany, France, Canada, UK, Italy, Mexico, Japan, Spain, Belgium, Korea Rep., Netherland } \\
\text { Hong Kong, Australia, Poland, Sweden, Austria, Turkey, Singapore }\end{array}$ \\
\hline 791 - Railway Vehicles/Equipmt & $\begin{array}{l}\text { Germany, USA, Canada, Russian Fed., UK, Switzerland, Netherlands, Austria, Kazakhstan, Italy, } \\
\text { Belgium, Mexico, Spain, China, Denmark, Greece, France, Czech Rep., Poland, Finland }\end{array}$ \\
\hline
\end{tabular}


The second approach for identifying the potential export markets for $\mathrm{B} \& \mathrm{H}$ products required examining the import volumes of countries in the world. The ranking of the import volumes of countries in the 2000-2004 periods revealed the countries with most potential demand. Among these markets there are some that B\&H products are not exported or exported on a very limited basis. These markets are good candidates to concentrate on with heavy promotion of $\mathrm{B} \& \mathrm{H}$ products with the purpose of establishing market share in the medium to long term. Entering these markets with highest demand is a strategy that B\&H exporters can utilize to strengthen their position in the world trade. Table 3 shows the list of these potential markets.

It should be noted that the strategy of entering the markets with highest import volumes can be a challenging one. The incumbent players in these markets with already established relationships or partnerships, difficulties in penetrating the distribution channels etc. are among such challenges. A more fruitful approach for $\mathrm{B} \& \mathrm{H}$ exporters, at least in the short term, might be to target the markets with the fastest growth of import volumes. The ranking of the countries according to their growth rates of import volume in the 2000-2005 periods include several developing countries that can offer many dynamic opportunities for B\&H exporters. These countries and their import volume growth rates are provided in Table 7.

Table 7: 40 Fastest Import Growth Countries

\begin{tabular}{|c|c|c|c|c|c|}
\hline Rank & Country & $\begin{array}{c}\text { Import Growth } \\
(2000-2005 \%)\end{array}$ & Rank & Country & $\begin{array}{c}\text { Import Growth } \\
(2000-2005 \%)\end{array}$ \\
\hline 1 & Afghanistan & 482 & 26 & Qatar & 147 \\
\hline 2 & Equatorial Guinea & 355 & 27 & Kuwait & 143 \\
\hline 3 & Sudan & 293 & 28 & Albania & 143 \\
\hline 4 & Georgia & 283 & 29 & Czech Republic & 140 \\
\hline 5 & Azerbaijan & 259 & 30 & Mauritania & 137 \\
\hline 6 & Kazakhstan & 244 & 31 & Viet Nam & 136 \\
\hline 7 & Ethiopia & 230 & 32 & Sierra Leone & 135 \\
\hline 8 & Congo & 226 & 33 & Croatia & 135 \\
\hline 9 & Serbia and Montenegro & 211 & 34 & Pakistan & 133 \\
\hline 10 & Romania & 210 & 35 & Niger & 133 \\
\hline 11 & Moldova & 198 & 36 & Bosnia and Herzegovina & 132 \\
\hline 12 & China & 193 & 37 & Bhutan & 128 \\
\hline 13 & Iran & 190 & 38 & Jordan & 127 \\
\hline 14 & Lithuania & 183 & 39 & South Africa & 124 \\
\hline 15 & Russian Federation & 180 & 40 & United Arab Emirates & 120 \\
\hline 16 & Bulgaria & 179 & 41 & Algeria & 119 \\
\hline 17 & Congo & 177 & 42 & Senegal & 119 \\
\hline 18 & Zambia & 177 & 43 & Turkey & 113 \\
\hline 19 & Slovak Republic & 177 & 44 & Mozambique & 109 \\
\hline 20 & Latvia & 169 & 45 & Marshall Islands & 106 \\
\hline 21 & Chad & 168 & 46 & Guinea-Bissau & 103 \\
\hline 22 & Angola & 168 & 47 & Swaziland & 100 \\
\hline 23 & Ukraine & 159 & 48 & Tajikistan & 97 \\
\hline 24 & Ecuador & 158 & 49 & Iraq & 69 \\
\hline 25 & India & 156 & 50 & Bolivarian Rep. of Venezuela & 54 \\
\hline
\end{tabular}

Source: International Trade Center (2006), ComTrade Data Set, http://www.intracen.org/tradstat/welcome.htm

One recent study that is valuable in identifying potential markets for B\&H exports is an International Trade Center working paper by Helmers and Pasteels (2005). They ran trade simulations using the TradeSim software to calculate (by comparing the predicted values to the observed trade numbers) trade potentials of developing countries. Their results for B\&H's potential export markets are provided in Table 8. "Untapped trade" and "highly untapped trade" countries (USA, Canada, India, Russia, many European countries) should be targeted by B\&H 
exporters in the 19 ISIC sectors (such as agricultural and hunting, forestry and fishing products, textiles, clothing and leather, motor vehicles and other transport equipment, precision equipment, etc.) included in the study. In the short run, B\&H exporters should try to keep their position in the countries with very strong and strong current trade.

Table 8: Trade Potentials for B\&H Exports

\begin{tabular}{|c|c|c|c|c|}
\hline Rank & Importer & $\begin{array}{c}\text { Current } \\
\text { Exports 2002- } \\
2003\end{array}$ & $\begin{array}{c}\text { Share in B\&H 's } \\
\text { exports of sector, in } \\
\%\end{array}$ & Relative Trade Potential \\
\hline 1 & Italy & 211,423 & 0.2 & Strong current trade (above predicted) \\
\hline 2 & Croatia & 189,321 & 0.179 & Very strong current trade \\
\hline 3 & Germany & 161,045 & 0.152 & Strong current trade (above predicted) \\
\hline 4 & Serbia & 112,198 & 0.106 & Very strong current trade \\
\hline 5 & Slovenia & 96,028 & 0.091 & Very strong current trade \\
\hline 6 & Switzerland & 83,499 & 0.079 & Very strong current trade \\
\hline 7 & Austria & 41,357 & 0.039 & Strong current trade (above predicted) \\
\hline 8 & France & 32,712 & 0.031 & High untapped trade potential \\
\hline 9 & United States & 12,016 & 0.011 & High untapped trade potential \\
\hline 10 & Macedonia & 9,251 & 0.009 & Very strong current trade \\
\hline 11 & Hungary & 8,300 & 0.008 & High untapped trade potential \\
\hline 12 & Belgium & 7,551 & 0.007 & High untapped trade potential \\
\hline 13 & Netherlands & 7,463 & 0.007 & High untapped trade potential \\
\hline 14 & Egypt & 7,368 & 0.007 & Very strong current trade \\
\hline 15 & Slovakia & 6,924 & 0.007 & Predicted = Current or low values \\
\hline 16 & Turkey & 6,367 & 0.006 & High untapped trade potential \\
\hline 17 & Spain & 5,932 & 0.006 & High untapped trade potential \\
\hline 18 & United Kingdom & 5,399 & 0.005 & High untapped trade potential \\
\hline 19 & Sweden & 4,867 & 0.005 & High untapped trade potential \\
\hline 20 & Greece & 4,819 & 0.005 & High untapped trade potential \\
\hline 21 & Poland & 4,620 & 0.004 & High untapped trade potential \\
\hline 22 & Czech Republic & 4,512 & 0.004 & High untapped trade potential \\
\hline 23 & $\begin{array}{c}\text { Libyan Arab } \\
\text { Jamahiriya }\end{array}$ & 2,719 & 0.003 & Very strong current trade \\
\hline 24 & Bulgaria & 2,324 & 0.002 & High untapped trade potential \\
\hline 25 & India & 2,158 & 0.002 & High untapped trade potential \\
\hline 26 & Congo & 2,126 & 0.002 & Very strong current trade \\
\hline 27 & Canada & 2,109 & 0.002 & High untapped trade potential \\
\hline 28 & Côte d'Ivoire & 1,434 & 0.001 & Very strong current trade \\
\hline 29 & $\begin{array}{c}\text { Russian } \\
\text { Federation }\end{array}$ & 1,343 & 0.001 & High untapped trade potential \\
\hline 30 & Tunisia & 1,303 & 0.001 & Predicted = Current or low values \\
\hline
\end{tabular}

Source: Christian Helmers and Jean-Michel Pasteels (2005), "TradeSim (third version), a gravity model for the calculation of trade potentials for developing countries and economies in transition”, International Trade Center Working Paper

The final approach for identifying the potential export markets for B\&H products is based on examining the import volumes at the product level. The products whose imports increased fastest in the 2000-2004 period and the countries that imported these products most are given in Table 9. By concentrating on the production of these products and targeting their destination countries as potential markets, $\mathrm{B} \& \mathrm{H}$ exporters may gain ground in the world trade. Based on the results of the section on the competitiveness of export products, the products B\&H exporters have high competitiveness in are not the same products with the highest import volume growth rates in the world. Hence, there is much room for planning and strategy making in this respect. 
Table 9: Fast Growing Import Products and Their Major Destinations

\begin{tabular}{|c|c|c|c|}
\hline $\begin{array}{l}\text { SITC } \\
\text { Code }\end{array}$ & Product & $\begin{array}{l}\text { 2000-2004 } \\
\text { \% Change }\end{array}$ & Importers \\
\hline 871 & Optical Instruments Nes. & 197 & $\begin{array}{l}\text { China, Japan, USA, Korea Rep., Hong, Kong, Mexico, Germany, Singapore, } \\
\text { France, United Kingdom, Spain, Brazil, Slovakia, Canada, Netherlands, Malaysia, } \\
\text { Italy }\end{array}$ \\
\hline 282 & Ferrous Waste/Scrap & 194 & $\begin{array}{l}\text { Korea Rep., China, Spain, Belgium, Germany, Italy, USA, India, Netherlands, } \\
\text { France, Finland, Luxembourg, Malaysia, Mexico, Indonesia }\end{array}$ \\
\hline 542 & Medicaments Include Vet. & 141 & $\begin{array}{l}\text { Belgium, USA, Germany, United Kingdom, France, Italy, Switzerland, } \\
\text { Netherlands, Spain, Canada, Japan, Australia, Russian, Fed., Greece, Poland, } \\
\text { Austria }\end{array}$ \\
\hline 281 & Iron Ore/Concentrates & 126 & $\begin{array}{l}\text { China, Japan, Germany, Korea Rep., Russian, Fed., Italy, France, Poland, United } \\
\text { Kingdom, Czech Rep., ., Netherlands, USA, Belgium, Slovakia, Austria, Canada, } \\
\text { Romania }\end{array}$ \\
\hline 671 & Pig Iron Etc Ferro Alloy & 118 & $\begin{array}{l}\text { USA, Japan, Korea Rep., Germany, Italy, China, Spain, Belgium, France, } \\
\text { Netherlands, Finland, Malaysia, United Kingdom, Mexico, Russian, Fed., Canada }\end{array}$ \\
\hline 321 & Coal Non-Agglomerated & 105 & $\begin{array}{l}\text { Japan, Korea Rep., United Kingdom, Germany, India, Italy, Spain, Netherlands, } \\
\text { France, USA, Brazil, Belgium, China, Canada, Israel, Finland, Denmark }\end{array}$ \\
\hline 287 & Base Metal Ore/Conc. Nes. & 104 & $\begin{array}{l}\text { China, Japan, Belgium, Korea Rep., USA, Netherlands, Germany, Spain, } \\
\text { Finland, Italy, Mexico, Canada, United Kingdom, France, Chile, Russia Fed., } \\
\text { Brazil, India }\end{array}$ \\
\hline 763 & Sound/Tv Recorders Etc. & 101 & $\begin{array}{l}\text { USA, Hong, Kong, Germany, Japan, United Kingdom, France, Netherlands, Spain, } \\
\text { Italy, Canada, China, Singapore, Australia, Belgium, Mexico, Korea Rep., Sweden }\end{array}$ \\
\hline 351 & Electric Current & 101 & $\begin{array}{l}\text { Austria, Italy, USA, Germany, France, Canada, Netherlands, United Kingdom, } \\
\text { Belgium, Hong, Kong, Norway, Sweden, Spain, Hungary, Finland, Denmark }\end{array}$ \\
\hline 422 & Fixed Veg. Oils Not Soft & 98 & $\begin{array}{l}\text { China, India, Netherlands, Germany, USA, Pakistan, Malaysia, United Kingdom, } \\
\text { Japan, Belgium, France, Italy, Bangladesh, Russia Fed., Egypt, Spain, Singapore }\end{array}$ \\
\hline 72 & Cocoa & 91 & $\begin{array}{l}\text { USA, Netherlands, Germany, France, Belgium, United Kingdom, Canada, } \\
\text { Malaysia, Russia Fed., Italy, Japan, Spain, Poland, Switzerland, Australia }\end{array}$ \\
\hline 283 & Copper Ores/Concentrates & 85 & $\begin{array}{l}\text { Japan, China, Korea Rep., Germany, India, Brazil, Spain, Finland, Bulgaria, } \\
\text { Philippines, Sweden, Canada, Zimbabwe, Russia Fed., Iran, Namibia, Oman, USA }\end{array}$ \\
\hline 335 & Residual Petrol. Prods & 85 & $\begin{array}{l}\text { Netherlands, USA, Belgium, China, Japan, Korea Rep., Germany, United } \\
\text { Kingdom, France, Italy, Spain, Malaysia, India, Mexico, Canada, Australia, } \\
\text { Ecuador, Israel }\end{array}$ \\
\hline 675 & Flat Rolled Alloy Steel & 82 & $\begin{array}{l}\text { China, Italy, Germany, France, USA, United Kingdom, Hong, Kong, Netherlands, } \\
\text { Mexico, Spain, Korea Rep., Canada, Belgium, Poland, Sweden, Thailand, Japan }\end{array}$ \\
\hline 761 & Television Receivers & 81 & $\begin{array}{l}\text { USA, United Kingdom, Germany, Netherlands, Italy, France, Japan, Hong, Kong, } \\
\text { Spain, Canada, Australia, Belgium, Sweden, Singapore, Mexico, Switzerland, } \\
\text { Austria }\end{array}$ \\
\hline 231 & Natural Rubber/Latex/Etc. & 80 & $\begin{array}{l}\text { USA, China, Japan, Korea Rep., Malaysia, Germany, France, Spain, Brazil, Italy, } \\
\text { Canada, Singapore, United Kingdom, Poland, Mexico, India }\end{array}$ \\
\hline 343 & Natural Gas & 79 & $\begin{array}{l}\text { USA, Germany, Japan, France, Belgium, Korea Rep., Spain, Ukraine, Netherlands, } \\
\text { Mexico, Canada, Hungary, Austria, Poland, United Kingdom, Slovakia, Belarus }\end{array}$ \\
\hline 812 & Sanitary/Plumb/Heat Fixt. & 78 & $\begin{array}{l}\text { United Kingdom, Germany, USA, France, Spain, Italy, Netherlands, Canada, } \\
\text { Belgium, Poland, Romania, Austria, Switzerland, Greece, Russia Fed., Czech Rep., } \\
\text { Ireland, Hungary }\end{array}$ \\
\hline 672 & Primary/Prods Iron/Steel & 75 & $\begin{array}{l}\text { USA, Korea Rep., Italy, China, Belgium, Germany, Thailand, Sweden, France, } \\
\text { Indonesia, Spain, Iran, Viet, Nam, United Kingdom, Egypt, Luxembourg, Morocco }\end{array}$ \\
\hline 541 & Pharmaceut. Exc Medicaments & 74 & $\begin{array}{l}\text { Germany, USA, France, Italy, Switzerland, Belgium, United Kingdom, } \\
\text { Netherlands, Japan, Spain, Canada, Austria, Brazil, Australia, Mexico, Ireland, } \\
\text { Korea Rep., China }\end{array}$ \\
\hline 676 & Iron/Steel Bars/Rods/Etc & 73 & $\begin{array}{l}\text { Germany, USA, France, Italy, Switzerland, Belgium, United Kingdom, } \\
\text { Netherlands, Japan, Spain, Canada, Austria, Brazil, Australia, Mexico, Ireland, } \\
\text { Korea Rep., China }\end{array}$ \\
\hline 111 & Beverage Non-Alcohol Nes. & 72 & $\begin{array}{l}\text { USA, United Kingdom, Germany, Belgium, France, Netherlands, Hong, Kong, } \\
\text { Japan, Canada, Spain, Italy, Ireland, Switzerland, Sweden, Austria, Luxembourg, } \\
\text { Singapore, Portugal }\end{array}$ \\
\hline 421 & Fixed Veg. Oil/Fat, Soft & 71 & $\begin{array}{l}\text { Italy, China, USA, India, France, Iran, Germany, Netherlands, Belgium, United } \\
\text { Kingdom, Spain, Japan, Mexico, Canada, Algeria, Korea Rep., Portugal, Morocco }\end{array}$ \\
\hline 793 & Ships/Boats/Etc & 70 & $\begin{array}{l}\text { Poland, Greece, USA, Germany, India, Italy, Spain, Denmark, France, Norway, } \\
\text { China, Korea Rep., Nigeria, Iran, Canada, Malaysia, Belgium }\end{array}$ \\
\hline 511 & Hydrocarbons/Derivatives & 70 & $\begin{array}{l}\text { China, Belgium, Germany, USA, Netherlands, Korea Rep., Mexico, France, } \\
\text { Indonesia, India, Spain, United Kingdom, Japan, Malaysia, Canada, Italy, } \\
\text { Singapore }\end{array}$ \\
\hline
\end{tabular}




\begin{tabular}{|c|c|c|c|}
\hline 872 & Medical/Etc Instruments & 69 & $\begin{array}{l}\text { USA, Germany, Japan, United Kingdom, Netherlands, France, Belgium, Italy, } \\
\text { Canada, Spain, Mexico, China, Australia, Hong, Kong, Switzerland, Singapore, } \\
\text { Sweden, Korea, Rep. }\end{array}$ \\
\hline 683 & Nickel & 68 & $\begin{array}{l}\text { USA, Germany, China, Japan, Netherlands, Korea Rep., Italy, United Kingdom, } \\
\text { Belgium, France, Sweden, Spain, Hong, Kong, Finland, India, South, Africa, } \\
\text { Brazil, Austria, Singapore }\end{array}$ \\
\hline 551 & Essent.Oil/Perfume/Flavr. & 68 & $\begin{array}{l}\text { USA, France, United Kingdom, Italy, Germany, Spain, Japan, Mexico, Canada, } \\
\text { Netherlands, Belgium, Poland, Switzerland, Ireland, Russia Fed., China, Indonesia, } \\
\text { Thailand }\end{array}$ \\
\hline 512 & Alcohols/Phenols/Derivs & 66 & $\begin{array}{l}\text { China, USA, Korea Rep., Germany, Japan, Netherlands, Italy, Belgium, France, } \\
\text { United Kingdom, Singapore, Thailand, India, Spain, Canada, Mexico, Indonesia, } \\
\text { Brazil }\end{array}$ \\
\hline 791 & Railway Vehicles/Equipmt. & 65 & $\begin{array}{l}\text { Germany, USA, Canada, Russia Fed., United Kingdom, Switzerland, Netherlands, } \\
\text { Austria, Kazakhstan, Italy, Belgium, Mexico, Spain, China, Denmark, Greece, } \\
\text { France, Czech Rep. }\end{array}$ \\
\hline 722 & Tractors & 63 & $\begin{array}{l}\text { USA, France, Canada, Spain, United Kingdom, Australia, Italy, Germany, } \\
\text { Belgium, Netherlands, Denmark, Norway, South, Africa, Poland, Austria, Japan, } \\
\text { Portugal, Sweden }\end{array}$ \\
\hline 553 & Perfume/Toilet/Cosmetics & 62 & $\begin{array}{l}\text { USA, United Kingdom, Germany, France, Japan, Spain, Italy, Canada, } \\
\text { Netherlands, Belgium, Russia Fed., Hong, Kong, Singapore, Switzerland, Ireland, } \\
\text { Australia, Mexico, Austria }\end{array}$ \\
\hline 723 & Civil Engineering Plant & 62 & $\begin{array}{l}\text { USA, Canada, France, China, United Kingdom, Germany, Singapore, Italy, Spain, } \\
\text { Netherlands, Belgium, Australia, Mexico, Austria, Indonesia, Russia Fed., India, } \\
\text { South, Africa }\end{array}$ \\
\hline 48 & Cereal Etc Flour/Starch & 62 & $\begin{array}{l}\text { USA, France, United Kingdom, Germany, Belgium, Canada, Netherlands, Japan, } \\
\text { Italy, Spain, Mexico, Austria, Ireland, Sweden, Denmark, Switzerland, Portugal, } \\
\text { Norway }\end{array}$ \\
\hline 73 & Chocolate/Cocoa Preps. & 62 & $\begin{array}{l}\text { France, USA, Germany, United Kingdom, Netherlands, Canada, Belgium, Japan, } \\
\text { Italy, Spain, Austria, Russia Fed., Sweden, Denmark, Mexico, Ireland, Portugal, } \\
\text { Czech Rep., }\end{array}$ \\
\hline 222 & Oil Seeds Etc - Soft Oil & 62 & $\begin{array}{l}\text { China, Japan, Netherlands, Germany, Mexico, Spain, Italy, Korea Rep., Belgium, } \\
\text { Thailand, Indonesia, United Kingdom, Portugal, USA, Canada, Malaysia, France }\end{array}$ \\
\hline 679 & Iron/Steel Pipe/Tube/Etc & 62 & $\begin{array}{l}\text { USA, Germany, China, France, Canada, United Kingdom, Italy, Spain, Norway, } \\
\text { Netherlands, Belgium, Russia Fed., Malaysia, Singapore, Mexico, Korea Rep., } \\
\text { Australia, Poland, Sweden }\end{array}$ \\
\hline 673 & Flat Rolled Iron/St Prod & 61 & $\begin{array}{l}\text { China, USA, Korea Rep., Italy, Germany, France, Spain, Belgium, Canada, Japan, } \\
\text { Thailand, United Kingdom, Netherlands, Poland, India, Mexico, Malaysia }\end{array}$ \\
\hline 899 & Misc. Manuf. Articles Nes. & 61 & $\begin{array}{l}\text { USA, Germany, France, Japan, United Kingdom, Netherlands, Italy, Hong, Kong, } \\
\text { Ireland, Spain, Switzerland, Canada, Belgium, China, Australia, Sweden, Korea } \\
\text { Rep., Austria }\end{array}$ \\
\hline 513 & Carboxylic Acid Compound & 59 & $\begin{array}{l}\text { China, USA, Belgium, Germany, Italy, France, United Kingdom, Netherlands, } \\
\text { Japan, Spain, Mexico, Canada, Korea Rep., Ireland, Switzerland, India, Brazil }\end{array}$ \\
\hline 721 & Agric Machine Ex Tractor & 58 & $\begin{array}{l}\text { USA, France, Germany, Canada, United Kingdom, Belgium, Spain, Australia, } \\
\text { Russia Fed., Netherlands, Denmark, Italy, Sweden, Austria, Mexico, Argentina, } \\
\text { Poland, Japan, Ireland, Switzerland, }\end{array}$ \\
\hline 971 & Gold Non-Monetary Ex Ore & 58 & $\begin{array}{l}\text { India, USA, Korea Rep., Italy, Untd. Arab. Em., Hong, Kong, Australia, Canada, } \\
\text { Malaysia, Japan, Germany, Thailand, Singapore, Spain, Pakistan, Saudi, Arabia, } \\
\text { France, Switzerland, Mexico, }\end{array}$ \\
\hline 24 & Cheese And Curd & 56 & $\begin{array}{l}\text { Germany, United Kingdom, Italy, USA, Belgium, France, Spain, Japan, } \\
\text { Netherlands, Russia Fed., Greece, Austria, Sweden, Saudi, Arabia, Switzerland, } \\
\text { Mexico, Denmark, Australia, Canada }\end{array}$ \\
\hline
\end{tabular}

Source: WTO (2004), Prepared from ComTrade Data Set, http://www.intracen.org/tradstat/welcome.htm

\section{Potential Products for B\&H Exporters}

In order for B\&H exporters achieve long term success in the world trade arena, it is crucial that they plan ahead utilizing carefully designed programs by the public sector, the private sector and the research institutions. The competitiveness of the B\&H export products can be improved by analyzing the potential success of each product/product group in foreign markets and then concentrating on promoting these products/products groups by using the resources of the country efficiently and effectively. 
With the purpose to help $\mathrm{B} \& \mathrm{H}$ exporters determine their directions in foreign markets, the products/product groups that have the highest potential for export success are identified. In this approach, most imported products as well as the products/product groups whose imports increased the fastest in the 2000-2004 period are examined. The results are provided in Table 10 and Table 9, respectively. Although this approach can be criticized because the products with the highest import volume or the fastest import growth in world markets are not necessarily the products that are suitable for B\&H exporters to specialize in, it is still possible to identify those products/product groups that present good opportunities to create or increase market shares in world exports given B\&H's resource strengths.

Table 10: Most Imported Products in the World

\begin{tabular}{|c|c|c|c|c|c|c|c|}
\hline Rank & $\begin{array}{c}\text { SITC } \\
\text { Code }\end{array}$ & Product & $\begin{array}{c}2000- \\
2004 \% \\
\text { Change }\end{array}$ & Rank & $\begin{array}{l}\text { SITC } \\
\text { Code }\end{array}$ & Product & $\begin{array}{c}2000- \\
2004 \% \\
\text { Change }\end{array}$ \\
\hline 1 & 333 & Petrol./Bitum. Oil,Crude & 42 & 23 & 893 & Articles Nes Of Plastics & 40 \\
\hline 2 & 781 & Passenger Cars Etc & 44 & 24 & 667 & Pearls/Precious Stones & 21 \\
\hline 3 & 776 & Valves/Transistors/Etc & 20 & 25 & 699 & Base Metal Manufac Nes & 37 \\
\hline 4 & 764 & Telecomms Equipment Nes & 25 & 26 & 851 & Footwear & 25 \\
\hline 5 & 752 & Computer Equipment & 21 & 27 & 684 & Aluminium & 33 \\
\hline 6 & 931 & Special Transactions Nes & 16 & 28 & 842 & Women/Girl Clothing Wven & 34 \\
\hline 7 & 784 & Motor Veh Parts/Access & 43 & 29 & 743 & Fans/Filters/Gas Pumps & 47 \\
\hline 8 & 334 & Heavy Petrol/Bitum Oils & 42 & 30 & 714 & Engines Non-Electric Nes & 12 \\
\hline 9 & 759 & Office Equip Parts/Accs. & 13 & 31 & 773 & Electrical Distrib Equip & 21 \\
\hline 10 & 542 & Medicaments Include Vet & 141 & 32 & 741 & Indust Heat/Cool Equipmt & 42 \\
\hline 11 & 778 & Electrical Equipment Nes & 27 & 33 & 541 & Pharmaceut Exc Medicamnt & 74 \\
\hline 12 & 772 & Electric Circuit Equipmt & 26 & 34 & 598 & Misc Chemical Prods Nes & 43 \\
\hline 13 & 792 & Aircraft/Spacecraft/Etc & 18 & 35 & 775 & Domestic Equipment & 52 \\
\hline 14 & 343 & Natural Gas & 79 & 36 & 841 & Mens/Boys Wear, Woven & 14 \\
\hline 15 & 874 & Measure/Control App Nes & 35 & 37 & 575 & Plastic Nes-Primary Form & 54 \\
\hline 16 & 713 & Internal Combust Engines & 38 & 38 & 763 & Sound/Tv Recorders Etc & 101 \\
\hline 17 & 845 & Articles Of Apparel Nes & 31 & 39 & 582 & Plastic Sheets/Film/Etc & 44 \\
\hline 18 & 641 & Paper/Paperboard & 24 & 40 & 771 & Elect Power Transm Equip & 11 \\
\hline 19 & 821 & Furniture/Stuff Furnishg & 50 & 41 & 57 & Fruit/Nuts, Fresh/Dried & 45 \\
\hline 20 & 728 & Special Indust Machn Nes & 26 & 42 & 898 & Musical Instrums/Records & 31 \\
\hline 21 & 782 & Goods/Service Vehicles & 34 & 43 & 716 & Rotating Electr Plant & 30 \\
\hline 22 & 515 & Organo-Inorganic Compnds & 42 & 44 & 673 & Flat Rolled Iron/St Prod & 61 \\
\hline
\end{tabular}

Source: WTO (2005), Prepared from ComTrade Data Set

Another approach to guide B\&H exporters in their efforts to improve their performance in foreign markets is to examine the import volumes of countries on a product basis. Ten countries with the highest total imports are examined to identify the products they import most as well as the products with the highest growth rate of imports (Table 11). A viable strategy for the B\&H exporters to establish themselves in these markets is to identify the products these markets demand most, or to identify those products whose demand in these markets grow the fastest, and concentrate their resources and efforts to produce and export these products. Similarly, it is also possible to identify the products/product groups whose import volumes are decreased the most between 2000 and 2004. This information can signal the businesses interested in improving their export competitiveness and performance the sectors they should pull out or avoid. 
Table 11: Top 5 Importers with Potential Products

USA

\begin{tabular}{|c|c|c|c|c|c|}
\hline Product & \begin{tabular}{|l} 
Total \\
Import
\end{tabular} & \begin{tabular}{|l|} 
Import \\
Growth
\end{tabular} & Product & Total Import & \begin{tabular}{|l|} 
Import \\
Growth
\end{tabular} \\
\hline 283 - Copper Ores/Concentrates & 216,006 & 3298.0 & 781 - Passenger Cars Etc & $575,995,120$ & 11.8 \\
\hline 551 - Essent.Oil/Perfume/Flavr & $4,853,766$ & 380.0 & 333 - Petrol./Bitum. Oil,Crude & $505,985,872$ & 52.4 \\
\hline 325 - Coke/Semi-Coke/Retort C & $2,447,733$ & 323.3 & 752 - Computer Equipment & $270,337,024$ & 6.5 \\
\hline 023 - Butter And Cheese & 411,730 & 275.2 & 931 - Special Transactions Nes & $245,985,296$ & 3.3 \\
\hline 047 - Cereal Meal/Flour N.E.S & 237,609 & 216.6 & 764 - Telecomms Equipment Nes & $214,367,856$ & 14.0 \\
\hline 282 - Ferrous Waste/Scrap & $2,962,987$ & 213.7 & 776 - Valves/Transistors/Etc & $161,820,992$ & -43.9 \\
\hline 245 - Fuel Wood/Wood Charcoal & 92,376 & 184.5 & 784 - Motor Veh Parts/Access & $160,311,600$ & 33.9 \\
\hline 542 - Medicaments Include Vet & $92,448,570$ & 170.0 & 334 - Heavy Petrol/Bitum Oils & $144,392,048$ & 45.2 \\
\hline 321 - Coal Non-Agglomerated & $3,967,633$ & 168.3 & 759 - Office Equip Parts/Accs. & $141,906,640$ & -10.8 \\
\hline 091 - Margarine/Shortening & 134,968 & 156.4 & 821 - Furniture/Stuff Furnishg & $121,633,728$ & 49.0 \\
\hline 269 - Worn Clothing Etc & 213,823 & 141.7 & 894 - Baby Carr/Toy/Game/Sport & $114,463,664$ & 12.8 \\
\hline 761 - Television Receivers & $54,247,747$ & 123.6 & 845 - Articles Of Apparel Nes & $113,865,952$ & 15.0 \\
\hline 883 - Cine Fild Developed & $1,068,865$ & 123.4 & 515 - Organo-Inorganic Compnds & $96,549,072$ & 23.8 \\
\hline 267 - Man-Made Fibres Nes/Wast & 265,753 & 121.8 & 542 - Medicaments Include Vet & $92,448,570$ & 170.0 \\
\hline 343 - Natural Gas & $84,403,796$ & 112.0 & 792 - Aircraft/Spacecraft/Etc & $91,125,277$ & -9.3 \\
\hline 674 - Rolled Plated M-Steel & $7,643,216$ & 110.1 & 343 - Natural Gas & $84,403,796$ & 112.0 \\
\hline 634 - Veneer/Plywood/Etc & $24,296,922$ & 106.3 & 782 - Goods/Service Vehicles & $84,215,522$ & 13.7 \\
\hline 342 - Liquid Propane/Butane & $10,320,766$ & 100.8 & 851 - Footwear & $81,647,205$ & 11.1 \\
\hline 872 - Medical/Etc Instruments & $34,703,434$ & 97.0 & 842 - Women/Girl Clothing Wven & $80,542,650$ & 15.9 \\
\hline 671 - Pig Iron Etc Ferro Alloy & $11,520,288$ & 93.4 & 713 - Internal Combust Engines & $80,038,822$ & 16.8 \\
\hline 016 - Meat/Offal Preserved & 755,841 & 93.0 & 772 - Electric Circuit Equipmt & $68,219,103$ & -3.1 \\
\hline 812 - Sanitary/Plumb/Heat Fixt & $3,640,008$ & 91.9 & 667 - Pearls/Precious Stones & $67,536,188$ & 18.5 \\
\hline 274 - Sulphur/Unroastd Pyrites & 319,181 & 85.2 & 874 - Measure/Control App Nes & $66,457,331$ & 20.8 \\
\hline
\end{tabular}

\section{Germany}

\begin{tabular}{|c|c|c|c|c|c|}
\hline Product & $\begin{array}{c}\text { Total } \\
\text { Import }\end{array}$ & $\begin{array}{l}\text { Import } \\
\text { Growth }\end{array}$ & Product & Total Import & $\begin{array}{l}\text { Import } \\
\text { Growth }\end{array}$ \\
\hline 883 - Cine Fild Developed & 27,799 & 342.2 & 931 - Special Transactions Nes & $218,074,204$ & 16.1 \\
\hline 325 - Coke/Semi-Coke/Retort C & $3,816,108$ & 273.3 & 781 - Passenger Cars Etc & $142,198,384$ & 70.1 \\
\hline 542 - Medicaments Include Vet & $56,132,032$ & 246.3 & 333 - Petrol./Bitum. Oil,Crude & $113,537,952$ & 41.0 \\
\hline 283 - Copper Ores/Concentrates & $2,672,659$ & 229.2 & 752 - Computer Equipment & $88,025,442$ & 20.7 \\
\hline 091 - Margarine/Shortening & 361,793 & 223.5 & 776 - Valves/Transistors/Etc & $70,350,646$ & 23.8 \\
\hline 282 - Ferrous Waste/Scrap & $3,843,682$ & 192.7 & 784 - Motor Veh Parts/Access & $70,306,839$ & 90.9 \\
\hline 677 - Iron/Steel Railway Matl & 694,551 & 177.0 & 792 - Aircraft/Spacecraft/Etc & $68,221,984$ & 39.2 \\
\hline 718 - Power Generating Equ Nes & $4,268,417$ & 172.9 & 764 - Telecomms Equipment Nes & $66,983,400$ & 69.8 \\
\hline 046 - Flour/Meal Wheat/Meslin & 124,519 & 156.1 & 343 - Natural Gas & $65,517,157$ & 108.2 \\
\hline 047 - Cereal Meal/Flour N.E.S & 75,993 & 151.1 & 542 - Medicaments Include Vet & $56,132,032$ & 246.3 \\
\hline 321 - Coal Non-Agglomerated & $6,420,655$ & 148.8 & 759 - Office Equip Parts/Accs. & $54,035,042$ & 33.4 \\
\hline 421 - Fixed Veg Oil/Fat, Soft & $1,989,823$ & 144.7 & 778 - Electrical Equipment Nes & $47,896,278$ & 33.1 \\
\hline 274 - Sulphur/Unroastd Pyrites & 34,807 & 139.0 & 713 - Internal Combust Engines & $45,415,981$ & 70.1 \\
\hline 763 - Sound/Tv Recorders Etc & $13,211,995$ & 138.2 & 334 - Heavy Petrol/Bitum Oils & $44,548,948$ & 9.4 \\
\hline 351 - Electric Current & $3,845,183$ & 133.8 & 641 - Paper/Paperboard & $37,109,512$ & 52.0 \\
\hline 541 - Pharmaceut Exc Medicamnt & $27,737,569$ & 131.2 & 821 - Furniture/Stuff Furnishg & $36,844,570$ & 43.3 \\
\hline 671 - Pig Iron Etc Ferro Alloy & $5,609,499$ & 129.8 & 772 - Electric Circuit Equipmt & $34,710,551$ & 49.2 \\
\hline 022 - Milk Pr Exc Buttr/Cheese & $5,082,006$ & 126.4 & 845 - Articles Of Apparel Nes & $34,672,044$ & 25.1 \\
\hline 061 - Sugar/Mollasses/Honey & $3,671,616$ & 121.5 & 874 - Measure/Control App Nes & $32,085,384$ & 46.2 \\
\hline 711 - Steam Generating Boilers & 337,368 & 110.2 & 714 - Engines Non-Electric Nes & $30,470,578$ & 5.9 \\
\hline 791 - Railway Vehicles/Equipmt & $4,204,236$ & 109.2 & 684 - Aluminium & $29,648,824$ & 42.0 \\
\hline 343 - Natural Gas & $65,517,157$ & 108.2 & 515 - Organo-Inorganic Compnds & $28,428,055$ & 6.0 \\
\hline 525 - Radio-Active Etc Matrial & $2,297,258$ & 107.9 & 541 - Pharmaceut Exc Medicamnt & $27,737,569$ & 131.2 \\
\hline 044 - Maize Except Sweet Corn. & $1,187,085$ & 105.3 & 842 - Women/Girl Clothing Wven & $26,424,579$ & 27.2 \\
\hline 579 - Plastic Waste/Scrap & 160,822 & 104.3 & 699 - Base Metal Manufac Nes & $25,841,673$ & 62.9 \\
\hline
\end{tabular}


China

\begin{tabular}{|c|c|c|c|c|c|}
\hline Product & \begin{tabular}{|l|} 
Total \\
Import
\end{tabular} & \begin{tabular}{|l|} 
Import \\
Growth
\end{tabular} & Product & Total Import & $\begin{array}{l}\text { Import } \\
\text { Growth }\end{array}$ \\
\hline $263-$ Cotton & $4,913,685$ & 2263.1 & 776 - Valves/Transistors/Etc & $206,913,072$ & 251.9 \\
\hline 871 - Optical Instruments Nes & $43,541,109$ & 2217.0 & 333 - Petrol./Bitum. Oil,Crude & $92,973,308$ & 128.2 \\
\hline 322 - Briquettes/Lignite/Peat & 26,417 & 2174.5 & 764 - Telecomms Equipment Nes & $80,967,894$ & 89.6 \\
\hline 289 - Precious Metal Ore/Conc. & 20,461 & 2087.2 & 759 - Office Equip Parts/Accs. & $50,857,973$ & 149.4 \\
\hline 677 - Iron/Steel Railway Matl & 154,416 & 1585.2 & 728 - Special Indust Machn Nes & $46,611,206$ & 160.9 \\
\hline 763 - Sound/Tv Recorders Etc & $2,407,742$ & 1317.3 & 772 - Electric Circuit Equipmt & $45,529,685$ & 175.5 \\
\hline 321 - Coal Non-Agglomerated & $1,739,764$ & 1200.0 & 871 - Optical Instruments Nes & $43,541,109$ & 2217.0 \\
\hline 671 - Pig Iron Etc Ferro Alloy & $2,290,992$ & 1140.3 & 752 - Computer Equipment & $42,097,974$ & 220.1 \\
\hline 041 - Wheat/Meslin & $2,088,380$ & 1013.1 & 874 - Measure/Control App Nes & $26,833,404$ & 196.9 \\
\hline 264 - Jute/Bast Fibre Raw/Retd & 33,718 & 989.3 & 334 - Heavy Petrol/Bitum Oils & $26,303,495$ & 152.6 \\
\hline 421 - Fixed Veg Oil/Fat, Soft & $3,617,380$ & 951.3 & 682 - Copper & $24,762,543$ & 120.7 \\
\hline 246 - Wood Chips/Waste & 93,564 & 771.2 & 673 - Flat Rolled Iron/St Prod & $24,744,408$ & 115.4 \\
\hline 223 - Oil Seeds-Not Soft Oil & 43,595 & 585.9 & 281 - Iron Ore/Concentrates & $24,684,867$ & 583.6 \\
\hline 284 - Nickel Ores/Concs/Etc & 233,551 & 584.2 & 222 - Oil Seeds Etc - Soft Oil & $21,482,277$ & 144.6 \\
\hline 281 - Iron Ore/Concentrates & $24,684,867$ & 583.6 & 784 - Motor Veh Parts/Access & $21,290,848$ & 245.0 \\
\hline 683 - Nickel & $2,801,643$ & 578.2 & 675 - Flat Rolled Alloy Steel & $20,233,215$ & 195.4 \\
\hline 781 - Passenger Cars Etc & $13,682,356$ & 504.3 & 792 - Aircraft/Spacecraft/Etc & $20,214,091$ & 129.9 \\
\hline 685 - Lead & 189,183 & 489.5 & 724 - Textile/Leather Machinry & $19,280,322$ & 115.8 \\
\hline 024 - Cheese And Curd & 45,016 & 452.4 & 513 - Carboxylic Acid Compound & $19,071,935$ & 192.7 \\
\hline 023 - Butter And Cheese & 54,719 & 413.3 & 575 - Plastic Nes-Primary Form & $18,762,502$ & 140.6 \\
\hline 287 - Base Metal Ore/Conc Nes & $5,081,730$ & 394.6 & 251 - Pulp And Waste Paper & $17,498,954$ & 97.7 \\
\hline 058 - Fruit Presvd/Fruit Preps & 121,398 & 393.0 & 641 - Paper/Paperboard & $17,468,360$ & 16.8 \\
\hline
\end{tabular}

France

\begin{tabular}{|c|c|c|c|c|c|}
\hline Product & \begin{tabular}{|l} 
Total \\
Import
\end{tabular} & \begin{tabular}{|l|} 
Import \\
Growth
\end{tabular} & Product & \begin{tabular}{|l|} 
Total \\
Import
\end{tabular} & $\begin{array}{l}\text { Import } \\
\text { Growth }\end{array}$ \\
\hline 286 - Uranium/Thorium Ore/Conc & 755 & 3625.0 & 781 - Passenger Cars Etc & $35,349,683$ & 53.4 \\
\hline 345 - Coal Gas/Water Gas/Etc & 1,152 & 3034.4 & 333 - Petrol./Bitum. Oil,Crude & $33,043,622$ & 38.8 \\
\hline 351 - Electric Current & $2,245,968$ & 248.4 & 752 - Computer Equipment & $32,624,884$ & 24.5 \\
\hline 325 - Coke/Semi-Coke/Retort C & $1,002,063$ & 199.7 & 784 - Motor Veh Parts/Access & $30,365,293$ & 84.9 \\
\hline 282 - Ferrous Waste/Scrap & $2,413,130$ & 159.3 & 542 - Medicaments Include Vet & $25,805,854$ & 112.1 \\
\hline 677 - Iron/Steel Railway Matl & 184,868 & 150.0 & 792 - Aircraft/Spacecraft/Etc & $25,120,889$ & 45.3 \\
\hline 044 - Maize Except Sweet Corn. & 640,444 & 141.6 & 764 - Telecomms Equipment Nes & $24,830,831$ & 13.9 \\
\hline 344 - Petrol./Hydrocarbon Gas & 149,940 & 135.6 & 334 - Heavy Petrol/Bitum Oils & $24,361,681$ & 65.3 \\
\hline 091 - Margarine/Shortening & 799,105 & 134.9 & 776 - Valves/Transistors/Etc & $23,144,643$ & -28.3 \\
\hline 899 - Misc Manuf Articles Nes & $9,020,396$ & 133.4 & 343 - Natural Gas & $22,979,338$ & 85.6 \\
\hline 793 - Ships/Boats/Etc & $5,183,698$ & 132.2 & 759 - Office Equip Parts/Accs. & $22,180,409$ & -7.7 \\
\hline 763 - Sound/Tv Recorders Etc & $8,742,035$ & 119.7 & 641 - Paper/Paperboard & $20,780,427$ & 28.4 \\
\hline 245 - Fuel Wood/Wood Charcoal & 80,235 & 113.2 & 845 - Articles Of Apparel Nes & $20,113,581$ & 52.6 \\
\hline 542 - Medicaments Include Vet & $38,369,062$ & 112.1 & 778 - Electrical Equipment Nes & $19,559,630$ & 28.6 \\
\hline 685 - Lead & 404,065 & 106.7 & 515 - Organo-Inorganic Compnds & $19,534,022$ & 21.9 \\
\hline 072 - Cocoa & $3,088,771$ & 104.3 & 714 - Engines Non-Electric Nes & $18,426,257$ & -15.4 \\
\hline 593 - Explosives/Pyrotechnics & 247,661 & 100.8 & 821 - Furniture/Stuff Furnishg & $17,580,272$ & 66.4 \\
\hline 718 - Power Generating Equ Nes & $3,334,437$ & 98.6 & 782 - Goods/Service Vehicles & $17,287,758$ & 70.1 \\
\hline 411 - Animal Oil/Fat & 299,812 & 90.6 & 713 - Internal Combust Engines & $17,266,451$ & 55.8 \\
\hline 871 - Optical Instruments Nes & $1,724,550$ & 90.0 & 893 - Articles Nes Of Plastics & $15,074,192$ & 51.5 \\
\hline 045 - Cereal Grains Nes & 81,750 & 87.4 & 874 - Measure/Control App Nes & $14,289,621$ & 51.6 \\
\hline 891 - Arms And Ammunition & 465,089 & 86.8 & 541 - Pharmaceut Exc Medicamnt & $13,764,424$ & 85.6 \\
\hline 246 - Wood Chips/Waste & 152,691 & 85.9 & 851 - Footwear & $13,376,349$ & 58.7 \\
\hline
\end{tabular}


England

\begin{tabular}{|c|c|c|c|c|c|}
\hline Product & \begin{tabular}{|l|} 
Total \\
Import \\
\end{tabular} & $\begin{array}{l}\text { Import } \\
\text { Growth }\end{array}$ & Product & \begin{tabular}{|l} 
Total \\
Import
\end{tabular} & $\begin{array}{l}\text { Import } \\
\text { Growth }\end{array}$ \\
\hline 325 - Coke/Semi-Coke/Retort C & 482,467 & 576.5 & 764 - Telecomms Equipment Nes & $66,530,705$ & 5.1 \\
\hline 343 - Natural Gas & $2,332,153$ & 430.0 & 784 - Motor Veh Parts/Access & $56,886,328$ & 50.2 \\
\hline 335 - Residual Petrol. Prods & $1,703,397$ & 232.9 & 333 - Petrol./Bitum. Oil,Crude & $47,533,733$ & 104.4 \\
\hline 675 - Flat Rolled Alloy Steel & $4,096,661$ & 212.6 & 542 - Medicaments Include Vet & $47,080,171$ & 130.7 \\
\hline 223 - Oil Seeds-Not Soft Oil & 141,470 & 196.4 & 759 - Office Equip Parts/Accs. & $45,999,238$ & -28.5 \\
\hline 883 - Cine Fild Developed & 58,594 & 170.7 & 776 - Valves/Transistors/Etc & $43,176,865$ & -30.8 \\
\hline 677 - Iron/Steel Railway Matl & 388,983 & 156.4 & 714 - Engines Non-Electric Nes & $32,741,440$ & 23.1 \\
\hline 025 - Eggs, Albumin & 522,018 & 150.8 & 667 - Pearls/Precious Stones & $29,318,949$ & 3.3 \\
\hline 812 - Sanitary/Plumb/Heat Fixt & $4,347,704$ & 146.6 & 641 - Paper/Paperboard & $29,055,460$ & 36.4 \\
\hline 122 - Tobacco, Manufactured & $1,508,109$ & 144.3 & 334 - Heavy Petrol/Bitum Oils & $28,507,836$ & 78.3 \\
\hline 321 - Coal Non-Agglomerated & $7,934,449$ & 140.8 & 874 - Measure/Control App Nes & $26,950,171$ & 19.4 \\
\hline 525 - Radio-Active Etc Matrial & $2,150,145$ & 137.0 & 845 - Articles Of Apparel Nes & $26,292,357$ & 69.4 \\
\hline 542 - Medicaments Include Vet & $47,080,171$ & 130.7 & 778 - Electrical Equipment Nes & $25,714,125$ & 8.7 \\
\hline 011 - Beef, Fresh/Chilld/Frozn & $3,758,447$ & 126.0 & 821 - Furniture/Stuff Furnishg & $24,888,604$ & 106.8 \\
\hline 872 - Medical/Etc Instruments & $10,299,488$ & 125.6 & 515 - Organo-Inorganic Compnds & $22,591,495$ & 124.0 \\
\hline 515 - Organo-Inorganic Compnds & $22,591,495$ & 124.0 & 112 - Alcoholic Beverages & $22,018,094$ & 55.3 \\
\hline 612 - Leather Manufactures & 537,166 & 114.9 & 842 - Women/Girl Clothing Wven & $19,954,031$ & 68.8 \\
\hline 763 - Sound/Tv Recorders Etc & $12,142,210$ & 113.7 & 894 - Baby Carr/Toy/Game/Sport & $19,713,687$ & 39.2 \\
\hline 811 - Prefabricated Buildings & 440,217 & 113.2 & 851 - Footwear & $18,508,019$ & 48.7 \\
\hline 722 - Tractors & $1,918,901$ & 112.1 & 782 - Goods/Service Vehicles & $18,394,890$ & 45.1 \\
\hline 761 - Television Receivers & $10,680,537$ & 109.4 & 772 - Electric Circuit Equipmt & $18,257,258$ & -8.5 \\
\hline
\end{tabular}

Source: WTO (2005), Prepared from ComTrade Data Set

Another very helpful tool to guide B\&H exporters comes from the above mentioned International Trade Center working paper by Helmers and Pasteels (2005) where they identified the sectors with "untapped trade potential" and "highly untapped trade potential" along with their respective markets based on 2002 and 2003 trade figures. These results are provided in Table 12.

Table 12: Relative Trade Potential: Country-Sector Pairs

\begin{tabular}{|c|l|l|c|c|}
\hline Rank & Importer & \multicolumn{1}{|c|}{ Sector } & $\begin{array}{c}\text { Current Exports } \\
\mathbf{2 0 0 2 - 2 0 0 3}\end{array}$ & Relative Trade Potential \\
\hline 1 & Italy & Chemicals and chemical products & 2,739 & Untapped trade potential \\
\hline 1 & Italy & Forestry and Fishing (products) & 2,677 & Untapped trade potential \\
\hline 3 & Germany & Wood and wood products & 8,641 & Untapped trade potential \\
\hline 3 & Germany & Electrical and electronic equipment & 2,104 & Untapped trade potential \\
\hline 6 & Switzerland & Textiles, clothing and leather & 1,658 & Untapped trade potential \\
\hline 7 & Austria & Metal and metal products & 4,817 & Untapped trade potential \\
\hline 7 & Austria & Textiles, clothing and leather & 3,430 & Untapped trade potential \\
\hline 8 & France & Metal and metal products & 7,148 & Untapped trade potential \\
\hline 8 & France & Electrical and electronic equipment & 410 & Untapped trade potential \\
\hline 9 & USA & Food, beverages and tobacco & 1,924 & Untapped trade potential \\
\hline 9 & USA & Precision instruments & 44 & Untapped trade potential \\
\hline 11 & Hungary & Machinery and equipment & 280 & Untapped trade potential \\
\hline 11 & Hungary & Electrical and electronic equipment & 25 & Untapped trade potential \\
\hline 12 & Belgium & Motor vehicles and other transport equipment & 203 & Untapped trade potential \\
\hline 12 & Belgium & Chemicals and chemical products & 121 & Untapped trade potential \\
\hline 13 & Netherlands & Electrical and electronic equipment & 458 & Untapped trade potential \\
\hline 13 & Netherlands & Motor vehicles and other transport equipment & 36 & Untapped trade potential \\
\hline 13 & Netherlands & Agriculture and hunting & 21 & Untapped trade potential \\
\hline 15 & Slovakia & Textiles, clothing and leather & 381 & Untapped trade potential \\
\hline 15 & Slovakia & Metal and metal products & 191 & Untapped trade potential \\
\hline 16 & Turkey & Motor vehicles and other transport equipment & 196 & Untapped trade potential \\
\hline 17 & Spain & Machinery and equipment & 443 & Untapped trade potential \\
\hline 17 & Spain & Chemicals and chemical products & 70 & Untapped trade potential \\
\hline
\end{tabular}




\begin{tabular}{|c|c|c|c|c|}
\hline 17 & Spain & Electrical and electronic equipment & 14 & Untapped trade potential \\
\hline 18 & UK & Machinery and equipment & 848 & Untapped trade potential \\
\hline 18 & UK & Electrical and electronic equipment & 260 & Untapped trade potential \\
\hline 19 & Sweden & Textiles, clothing and leather & 1,484 & Untapped trade potential \\
\hline 19 & Sweden & Motor vehicles and other transport equipment & 109 & Untapped trade potential \\
\hline 20 & Greece & Machinery and equipment & 142 & Untapped trade potential \\
\hline 20 & Greece & Motor vehicles and other transport equipment & 57 & Untapped trade potential \\
\hline 21 & Poland & Food, beverages and tobacco & 68 & Untapped trade potential \\
\hline 21 & Poland & Motor vehicles and other transport equipment & 56 & Untapped trade potential \\
\hline 22 & Czech Rep. & Wood and wood products & 441 & Untapped trade potential \\
\hline 22 & Czech Rep. & Textiles, clothing and leather & 220 & Untapped trade potential \\
\hline 22 & Czech Rep. & Other manufacturing & 194 & Untapped trade potential \\
\hline 24 & Bulgaria & Metal and metal products & 39 & Untapped trade potential \\
\hline 24 & Bulgaria & Wood and wood products & 32 & Untapped trade potential \\
\hline 24 & Bulgaria & Textiles, clothing and leather & 8 & Untapped trade potential \\
\hline 25 & India & Metal and metal products & 184 & Untapped trade potential \\
\hline 27 & Canada & Metal and metal products & 109 & Untapped trade potential \\
\hline 27 & Canada & Wood and wood products & 11 & Untapped trade potential \\
\hline 29 & Russian Fed. & Textiles, clothing and leather & 96 & Untapped trade potential \\
\hline 29 & Russian Fed. & Metal and metal products & 58 & Untapped trade potential \\
\hline 29 & Russian Fed. & Food, beverages and tobacco & 17 & Untapped trade potential \\
\hline 1 & Italy & Motor vehicles and other transport equipment & 1,033 & High untapped trade potential \\
\hline 3 & Germany & Metal and metal products & 15,007 & High untapped trade potential \\
\hline 3 & Germany & Food, beverages and tobacco & 2,114 & High untapped trade potential \\
\hline 6 & Switzerland & Other manufacturing & 126 & High untapped trade potential \\
\hline 8 & France & Textiles, clothing and leather & 5,272 & High untapped trade potential \\
\hline 8 & France & Machinery and equipment & 868 & High untapped trade potential \\
\hline 8 & France & Wood and wood products & 644 & High untapped trade potential \\
\hline 9 & USA & Other manufacturing & 4,894 & High untapped trade potential \\
\hline 9 & USA & Textiles, clothing and leather & 2,134 & High untapped trade potential \\
\hline 9 & USA & Machinery and equipment & 1,349 & High untapped trade potential \\
\hline 9 & USA & Metal and metal products & 689 & High untapped trade potential \\
\hline 9 & USA & Motor vehicles and other transport equipment & 239 & High untapped trade potential \\
\hline 9 & USA & Wood and wood products & 115 & High untapped trade potential \\
\hline 9 & USA & Chemicals and chemical products & 94 & High untapped trade potential \\
\hline 9 & USA & Electrical and electronic equipment & 64 & High untapped trade potential \\
\hline 11 & Hungary & Textiles, clothing and leather & 640 & High untapped trade potential \\
\hline 11 & Hungary & Metal and metal products & 428 & High untapped trade potential \\
\hline 11 & Hungary & Wood and wood products & 178 & High untapped trade potential \\
\hline 12 & Belgium & Wood and wood products & 579 & High untapped trade potential \\
\hline 12 & Belgium & Metal and metal products & 341 & High untapped trade potential \\
\hline 12 & Belgium & Other manufacturing & 83 & High untapped trade potential \\
\hline 13 & Netherlands & Metal and metal products & 2,959 & High untapped trade potential \\
\hline 13 & Netherlands & Wood and wood products & 958 & High untapped trade potential \\
\hline 13 & Netherlands & Food, beverages and tobacco & 404 & High untapped trade potential \\
\hline 13 & Netherlands & Textiles, clothing and leather & 132 & High untapped trade potential \\
\hline 16 & Turkey & Textiles, clothing and leather & 82 & High untapped trade potential \\
\hline 16 & Turkey & Wood and wood products & 50 & High untapped trade potential \\
\hline 16 & Turkey & Metal and metal products & 36 & High untapped trade potential \\
\hline 17 & Spain & Metal and metal products & 2,436 & High untapped trade potential \\
\hline 17 & Spain & Wood and wood products & 1,667 & High untapped trade potential \\
\hline 17 & Spain & Food, beverages and tobacco & 229 & High untapped trade potential \\
\hline 17 & Spain & Textiles, clothing and leather & 215 & High untapped trade potential \\
\hline 17 & Spain & Other manufacturing & 81 & High untapped trade potential \\
\hline 17 & Spain & Motor vehicles and other transport equipment & 18 & High untapped trade potential \\
\hline 18 & UK & Other manufacturing & 1,579 & High untapped trade potential \\
\hline 18 & UK & Wood and wood products & 766 & High untapped trade potential \\
\hline 18 & UK & Metal and metal products & 470 & High untapped trade potential \\
\hline 18 & UK & Textiles, clothing and leather & 324 & High untapped trade potential \\
\hline 18 & UK & Food, beverages and tobacco & 140 & High untapped trade potential \\
\hline 18 & UK & Motor vehicles and other transport equipment & 100 & High untapped trade potential \\
\hline 19 & Sweden & Metal and metal products & 23 & High untapped trade potential \\
\hline
\end{tabular}




\begin{tabular}{|c|l|l|c|c|}
\hline 20 & Greece & Wood and wood products & 520 & High untapped trade potential \\
\hline 20 & Greece & Food, beverages and tobacco & 67 & High untapped trade potential \\
\hline 20 & Greece & Textiles, clothing and leather & 346 & High untapped trade potential \\
\hline 21 & Poland & Wood and wood products & 61 & High untapped trade potential \\
\hline 21 & Poland & Metal and metal products & 48 & High untapped trade potential \\
\hline 21 & Poland & Textiles, clothing and leather & 1,177 & High untapped trade potential \\
\hline 22 & Czech Republic & Metal and metal products &
\end{tabular}

Source: Christian Helmers and Jean-Michel Pasteels (2005), "TradeSim (third version), a gravity model for the calculation of trade potentials for developing countries and economies in transition", International Trade Center Working Paper

\section{CONCLUSION}

The purpose of this study is to provide B\&H exporters a starting point in evaluating their product and market selection strategies. Long term sustainable success in the ever changing global economy requires a close and continuous scanning of the trends in the environment. The trends in the export volumes of various products/product groups and their destination countries include important clues for B\&H exporters in terms of shaping their future strategies aimed at sustaining or improving their competitiveness in foreign markets. Same information is also crucial for the B\&H government in designing a successful industrial development program as well as an export incentives scheme. For example, based on the analyses in the previous sections, there is major trade potential between B\&H other European countries in a variety of industries (wood and wood products, motor vehicles, textiles etc.). An industrial program that fosters the development of any of these industries given the resources of B\&H and carefully designed export incentives would benefit the individual exporter firms and the country in general.

\section{REFERENCES}

1. Helmers, Christian and Jean-Michel Pasteels (2005), TradeSim (third version), a gravity model for the calculation of trade potentials for developing countries and economies in transition, International Trade Center Working Paper

2. International Trade Center (2006), ComTrade Data Set, http://www.intracen.org/tradstat/welcome.htm

3. Link2Exports (2007), Bosnia and Herzegovina, http://www.link2exports.co.uk/regions.asp?1sid=1418\&pid=1213

4. $\quad$ UNDP (1998), Human Development Report - Bosnia and Herzegovina. Sarajevo

5. UNDP (2003). Bosnia and Herzegovina Human Development Report/Millennium Development Goals 2003, Sarajevo

6. World Bank (2000), Bosnia and Herzegovina - Country Assistance Strategy, South East Europe Country Unit (ECCU4), Report \# 20592, Washington DC

7. World Bank (2005), Doing Business in 2006: Creating Jobs, Washington DC

8. World Bank (2005), Enterprise Surveys, http://www.enterprisesurveys.org/ExploreEconomies/Default.aspx?economyid=26.

9. WTO (2004), World Trade Report 2004: Exploring the Linkage Between the Domestic Policy Environment and International Trade, WTO, Geneva

10. WTO (2005), World Trade Report 2005. Exploring the links between trade, standards and the WTO, Geneva 


\section{NOTES}

